July 1994

mall

UMDEPP 95-06

\title{
Why Are There So Many $N=4$ Superstrings?
}

\author{
S. James Gates, Jr. 1, 2 \\ Department of Physics \\ University of Maryland \\ College Park, MD 20742-4111 USA \\ gates@umdhep.umd.edu
}

\begin{abstract}
We demonstrate the existence of three off-shell distinct $\mathrm{N}=4$ superstrings at the level of manifest locally supersymmetric Lagrangian field theories on the world sheet.
\end{abstract}

\footnotetext{
${ }^{1}$ Supported by National Science Foundation Grant PHY-91-19746

${ }^{2}$ Supported by NATO Grant CRG-93-0789
} 


\section{Introduction}

There are three $2 \mathrm{D}, \mathrm{N}=4$ off-shell scalar multiplets with a finite number of auxiliary fields. This is a little known fact that is the primary motivation for this presentation. In the following we will give, to our knowledge, the first comprehensive discussion regarding this curious situation. The fact that this situation exist at all is extremely puzzling. There is a known precedent for this situation. The resolution of the puzzle in the precedent $\mathrm{N}=2$ models [1] resides in the existence of "mirror symmetry." The four distinct off-shell $\mathrm{N}=2$ models pointed out in reference [1] are related pair-wise by a mirror transformation that replaces chiral superfields by twisted chiral superfields. The existence of mirror symmetry, at least at the level of lagrangian field theories, is a consequence that follows since both chiral and twisted chiral multiplets [2] exist as distinct off-shell $\mathrm{N}=2$ scalar multiplet representations. As we argued, $\mathrm{N}=2$ superstrings can be constructed utilizing two chiral multiplets, two anti-chiral multiplets and one chiral multiplet and one twisted chiral multiplet. Interestingly enough, models of this last type have recently appeared in the context of exact solutions for "stringy" plane waves [3].

For a long time, issues regarding $\mathrm{N}=4$ superstrings have simply not seemed pressing. Apart from the original work discovering these theories [4], there has been a little work carried out on their quantization [5]. This was largely due to the belief that the critical dimension of such theories was minus one. This "standard folklore" has recently been challanged [6], with the proposal that the actual critical value is plus one! Even should this new proposal not stand the test of time, the recent new insight into integrable supersymmetric models that can be understood as string theories away from their critical dimensions suggest that there is reason to believe that interesting systems may very well be described by $\mathrm{N}=4$ superstring theories. Fortunately, we are very well situated to study $\mathrm{N}=4$ superstrings. Off-shell structures have been known for some time for $2 \mathrm{D}, \mathrm{N}=4$ supergravity [1, 7].

\section{Type-I and Type-II, $\mathrm{N}=4$ Superstrings}

These are theories of $\mathrm{N}=4$ strings that are based on twisted $\mathrm{N}=4$ scalar multiplets. These types of scalar multiplets may be regarded as having origins as dimensional reductions from 3D or 4D. There are $8-8$ fermion and boson degrees of freedom in these scalar multiplets. 
We start with a $4 \mathrm{D}, \mathrm{N}=2$ vector multiplet and a $4 \mathrm{D}, \mathrm{N}=2$ tensor multiplet. Considering a compactification to 3D splits off some of the components of the gauge fields in each multiplet into different 3D representations of the $\mathrm{SO}(1,2)$ group. The $4 \mathrm{D}$ vector gauge field "yields" a 3D vector gauge field and one scalar. Similarly, a 4D, 2-form gauge field "yields" a 3D, 2-form gauge field and a 3D vector gauge field. In $3 \mathrm{D}$, by a duality transformation, the former can be replaced by an auxiliary scalar. Since an irreducible spinor representation of $\mathrm{SO}(1,3)$ contains two irreducible spinor representations of $\mathrm{SO}(1,2)$ The number of supersymmetries for the multiplets double. So the multiplets that result are $3 \mathrm{D}, \mathrm{N}=4$ representations. We conclude there may exist two distinct 3D, $\mathrm{N}=4$ vector multiplets 3 . These two different representations of the same physical degrees of freedom are an example of the occurence of variant superfield representations [9].

Now we continue from $3 \mathrm{D}$ to $2 \mathrm{D}$ via a further compactification. This time the 3D vector gauge field in each multiplet "yields" a 2D vector gauge field as well as one scalar. In 2D, a gauge vector propagates no physical degrees of freedom and by a duality transformation can be replaced by an auxiliary scalar. The $4 \mathrm{D}, \mathrm{N}=2$ vector multiplet reduces to a $2 \mathrm{D}, \mathrm{N}=4$ scalar multiplet (called the twisted-I multiplet) and the $4 \mathrm{D}, \mathrm{N}=2$ tensor multiplet also reduces to a $2 \mathrm{D}, \mathrm{N}=4$ scalar multiplet (called the twisted-II multiplet). The explicit form of these two $2 \mathrm{D}, \mathrm{N}=4$ twisted scalar multiplets are given below.

The twisted-I multiplet has been discussed several times before [1, 2]. The supersymmetry variations are characterized by,

$$
\begin{aligned}
D_{\alpha i} F= & 2 C_{i j} \psi_{\alpha}{ }^{j}, \\
\bar{D}_{\alpha}{ }^{i} F= & 0 \\
D_{\alpha i} S= & -i \bar{\psi}_{\alpha i}, \\
D_{\alpha i} P= & \left(\gamma^{3}\right)_{\alpha}{ }^{\beta} \bar{\psi}_{\beta i}, \\
D_{\alpha i} \psi^{\beta j}= & \delta_{i}{ }^{j}\left[\left(\gamma^{c}\right)_{\alpha}{ }^{\beta}\left(\partial_{c} S\right)+i\left(\gamma^{3} \gamma^{c}\right)_{\alpha}{ }^{\beta}\left(\partial_{c} P\right)\right] \\
& +\frac{1}{2}\left[\delta_{i}{ }^{j}\left(\gamma^{3}\right)_{\alpha}{ }^{\beta} A+i \delta_{\alpha}{ }^{\beta} A_{i}{ }^{j}\right], \\
\bar{D}_{\alpha}{ }^{i} \psi^{\beta j}= & i C^{i j}\left(\gamma^{c}\right)_{\alpha}{ }^{\beta}\left(\partial_{c} F\right), \\
D_{\alpha i} A= & -i 2\left(\gamma^{3} \gamma^{c}\right)_{\alpha}{ }^{\beta} \partial_{c} \bar{\psi}_{\beta i}, \\
D_{\alpha i} A_{j}{ }^{k}= & 4\left(\delta_{j}{ }^{l} \delta_{i}{ }^{k}-\frac{1}{2} \delta_{j}{ }^{k} \delta_{i}{ }^{l}\right)\left(\gamma^{c}\right)_{\alpha}{ }^{\beta} \partial_{c} \bar{\psi}_{\beta l},
\end{aligned}
$$

With the exception of $F$ and $\psi_{\alpha i}$, all the remaining fields are real (for $\left.A_{i}{ }^{j}=\left(A_{j}{ }^{i}\right)^{*}\right)$.

\footnotetext{
${ }^{3}$ The existence of these two distinct $3 \mathrm{D}, \mathrm{N}=4$ vector multiplets plays a very important role in topological field theory [8].
} 
The invariant component level action takes the explicit form,

$$
\begin{aligned}
\mathcal{S}_{\mathrm{TM}-\mathrm{I}, \mathrm{N}=4}=\int d^{2} \sigma\left[\frac{1}{2} S \square S\right. & +\frac{1}{2} P \square P+\frac{1}{2} F \square \bar{F}+i \psi^{\alpha i}\left(\gamma^{c}\right)_{\alpha \beta} \partial_{c} \bar{\psi}^{\beta}{ }_{i} \\
& \left.-\frac{1}{4} A^{2}-\frac{1}{16} A_{i}{ }^{j} A_{j}{ }^{i}\right] .
\end{aligned}
$$

In the presence of $2 \mathrm{D}, \mathrm{N}=4$ supergravity, we can write an action for this multiplet if we introduce two pre-potential superfields; $V$ and $V_{i}{ }^{j}$. The highest components of $V$ and $V_{i}{ }^{j}$ correspond to $A$ and $A_{i}{ }^{j}$, respectively. The resulting expression for the action describes the twisted-I, $\mathrm{N}=4$ superstring prior to any gauge fixing.

$$
\mathcal{S}_{\mathrm{TM}-\mathrm{I}, \mathrm{N}=4}=-\int d^{2} \sigma d^{8} \theta E^{-1}\left[V A+V_{i}^{j} A_{j}^{i}\right] .
$$

The twisted-II theory was first found by Ivanov and Krivonos [10]. A description that is equivalent to their previous work is given by,

$$
\begin{aligned}
D_{\alpha i} \mathcal{T}= & \left(\gamma^{3}\right)_{\alpha}{ }^{\beta} \Psi_{\beta i}, \\
D_{\alpha i} \mathcal{X}_{j}{ }^{k}= & i\left[\delta_{i}{ }^{k} \Psi_{\alpha j}-\frac{1}{2} \delta_{j}{ }^{k} \Psi_{\alpha i}\right], \\
\mathcal{X}_{i}{ }^{i}= & 0, \mathcal{X}_{i}{ }^{j}-\left(\mathcal{X}_{j}{ }^{i}\right)^{*}=0, \\
D_{\alpha i} \Psi_{\beta j}= & \frac{1}{2} C_{\alpha \beta} C_{i j} \bar{J}, \\
D_{\alpha i} \bar{J}= & 0, m-(m)^{*}=0, n-(n)^{*}=0 \\
\bar{D}_{\alpha}{ }^{i} \Psi_{\beta j}= & i \delta_{j}{ }^{i}\left(\gamma^{3} \gamma^{a}\right)_{\alpha \beta}\left(\partial_{a} \mathcal{T}\right)+2\left(\gamma^{a}\right)_{\alpha \beta}\left(\partial_{a} \mathcal{X}_{j}{ }^{i}\right) \\
& +i \frac{1}{2} C_{\alpha \beta} \delta_{j}{ }^{i} m+\frac{1}{2}\left(\gamma^{3}\right)_{\alpha \beta} \delta_{j}{ }^{i} n . \\
D_{\alpha i} J= & -i 4 C_{i j}\left(\gamma^{a}\right)_{\alpha}{ }^{\beta}\left(\partial_{a} \bar{\Psi}_{\beta}{ }^{j}\right) \\
D_{\alpha i} n= & -i 2\left(\gamma^{3} \gamma^{a}\right)_{\alpha}{ }^{\beta}\left(\partial_{a} \Psi_{\beta i}\right), \\
D_{\alpha i} m= & -2\left(\gamma^{a}\right)_{\alpha}{ }^{\beta}\left(\partial_{a} \Psi_{\beta i}\right) .
\end{aligned}
$$

Here the complex fields are $J$ and $\Psi_{\alpha i}$.

An invariant component level action takes the explicit form,

$$
\begin{gathered}
\mathcal{S}_{\mathrm{TM}-\mathrm{II}, \mathrm{N}=4}=\int d^{2} \sigma\left[\frac{1}{2} \mathcal{T} \square \mathcal{T}+\mathcal{X}_{j}{ }^{i} \square \mathcal{X}_{i}{ }^{j}+i \Psi^{\alpha}{ }_{i}\left(\gamma^{c}\right)_{\alpha \beta} \partial_{c} \bar{\Psi}^{\beta i}\right. \\
\left.-\frac{1}{8}\left(m^{2}+n^{2}+J \bar{J}\right)\right] .
\end{gathered}
$$

In the presence of $2 \mathrm{D}, \mathrm{N}=4$ supergravity, we can write an action for this multiplet if we introduce three pre-potential superfields; $K, L$ and $\Lambda$ (with $\bar{D}_{\alpha}{ }^{i} \Lambda=0$ ). The 
highest components of $K, L$ and $\Lambda$ correspond to $m, n$ and $J$, respectively. The resulting expression for the action describes the twisted-II, $\mathrm{N}=4$ superstring prior to any gauge fixing,

$$
\begin{aligned}
\mathcal{S}_{\mathrm{TM}-\mathrm{II}, \mathrm{N}=4}=- & \int d^{2} \sigma d^{8} \theta E^{-1}[K m+L n] \\
& -\int d^{2} \sigma d^{4} \theta \mathcal{E}^{-1} \Lambda J-\int d^{2} \sigma d^{4} \bar{\theta} \overline{\mathcal{E}}^{-1} \bar{\Lambda} \bar{J}
\end{aligned}
$$

Here $\mathcal{E}^{-1}$ and $\overline{\mathcal{E}}^{-1}$ denote chiral and anti-chiral density measures.

Even in 2D these two multiplets possess a "duality." In terms of equations, this duality is made explicit by observing,

$$
\begin{array}{lll}
D_{\alpha i} F+i 2 C_{i j} \bar{D}_{\alpha}{ }^{j} S=0 \quad, \quad \bar{D}_{\alpha}{ }^{i} F=0 \quad, & D_{\alpha i} S+i\left(\gamma^{3}\right)_{\alpha}{ }^{\beta} D_{\beta i} P=0 \quad, \\
D_{\alpha i} J+i 2 C_{i j} \bar{D}_{\alpha}{ }^{j} m=0 \quad, & \bar{D}_{\alpha}{ }^{i} J=0 \quad, \quad D_{\alpha i} m+i\left(\gamma^{3}\right)_{\alpha}{ }^{\beta} D_{\beta i} n=0
\end{array} .
$$

That is, we can define a duality map that interchanges the physical spin-0 fields in one of the twisted multiplets with the auxiliary spin-0 fields in the other one of the twisted multiplets and vice-versa.

The most powerful manifestation of this phenomena is the existence of an $\mathrm{N}=4$ mass term! Previously the only known way to introduce such mass terms in $2 \mathrm{D}, \mathrm{N}=$ 4 theories required the use of "active central charges" that was discussed a very long time ago [11]. Since these two dual $N=4$ scalar multiplets exist, there is a much simpler way to construct massive $2 \mathrm{D}, \mathrm{N}=4$ representations. This mass is produced in "associated production." That is, a Twisted-I multiplet and a Twisted-II multiplet simultaneously acquire mass. This phenomena of the associated production of mass has been observed before [12] in the context of $4 \mathrm{D}, \mathrm{N}=1$ theories. Here it is easy to show that the following action is invariant under the supersymmetry variations described above.

$$
\begin{aligned}
\mathcal{S}_{\mathrm{N}=4, \text { mass }}=\int d^{2} \sigma M_{0}[ & \frac{1}{2} m S-\frac{1}{2} n P-\frac{1}{4}(J \bar{F}+\bar{J} F) \\
& \left.-\frac{1}{8} \mathcal{X}_{i}{ }^{j} A_{j}{ }^{i}-\frac{1}{2} \mathcal{T} A+\left(\bar{\Psi}^{\alpha i} \bar{\psi}_{\alpha i}+\text { h.c. }\right)\right] .
\end{aligned}
$$

We should mention perhaps that this mass term is to be expected from the point of view of a recently derived result for $3 \mathrm{D}, \mathrm{N}=4 \mathrm{BF}$ theory 8$]$.

\section{The HM-II, $\mathrm{N}=4$ Superstring}

Some time ago, an off-shell scalar multiplet (called the "relaxed hypermultiplet")

with manifest $4 \mathrm{D}, \mathrm{N}=2$ supersymmetry was found 13 . This led to the discovery of 
a whole previously unknown class of finite $\mathrm{N}=2$ supersymmetric Yang-Mills-matter models [14]. This off-shell representation also gives a very simple tool to derive yet one more off-shell formulation of a superstring with $2 \mathrm{D}, \mathrm{N}=4$ supersymmetry. Only a simple toroidal compactification of the $4 \mathrm{D}$ action is required! The number of components in this scalar multiplet is far greater than in the twisted type-I and typeII twisted scalar multiplets. It has 32-32 fermionic and bosonic degrees of freedom. We will call the resultant $N=4$ superstring the HM-II, $N=4$ superstring. Since the number of fields is so large, it is simplest to begin with its superspace action.

$$
\mathcal{S}_{\mathrm{HM}, \mathrm{N}=4}=\int d^{2} \sigma d^{8} \theta E^{-1}\left[\left(\lambda_{\alpha}{ }^{i} \rho_{i}^{\alpha}+\bar{\lambda}_{\alpha i} \bar{\rho}^{\alpha i}\right)+L^{i j k l} X_{i j k l}\right] .
$$

In this expression the fundamentally unconstrained superfield potentials are $\rho^{\alpha}{ }_{i}$ and $X_{i j k l}$ (with $X_{i j k l}=C_{i i^{\prime}} C_{j j^{\prime}} C_{k k^{\prime}} C_{l l^{\prime}} X^{i^{\prime} j^{\prime} k^{\prime} l^{\prime *}}$ ). In this action, the superdeterminant density is that associated with the minimal $\mathrm{N}=4$ supergravity theory described in [1].

In order to describe this theory at the level of components, we simply "borrow" the results from reference [13]. However, we first make certain field re-definitions that "diagonalize" the component action and result in the obvious separation of auxiliary fields from propagating fields. These re-definitions take the forms,

$$
\begin{gathered}
\varphi \equiv[V] \quad, \quad \varphi_{i j} \equiv 2\left[L_{i j}\right] \quad, \quad \psi^{\alpha i} \equiv \frac{4}{3}\left[\lambda^{\alpha i}\right], \\
\lambda^{\alpha i} \equiv\left[\psi^{\alpha i}\right]-\frac{2}{3}\left[\lambda^{\alpha i}\right], \chi_{\alpha i} \equiv\left[\xi_{\alpha i}\right], \lambda^{\alpha i j k} \equiv\left[\psi^{\alpha i j k}\right], \chi^{\alpha}{ }_{i j k} \equiv\left[\xi^{\alpha}{ }_{i j k}\right]-i \frac{8}{3} \partial^{\alpha \beta}\left[\bar{\psi}_{\beta i j k}\right] \\
C^{i j k l} \equiv\left[C^{i j k l}\right], L_{i j k l} \equiv\left[L_{i j k l}\right], M^{i j} \equiv\left[K^{i j}\right]-\frac{3}{2}\left[M^{i j}\right], \quad N \equiv[N] \\
K^{i j} \equiv\left[K^{i j}\right]+\frac{1}{2}\left[M^{i j}\right], G_{\alpha \beta} \equiv\left[G_{\alpha \beta}\right]+6 \partial_{\alpha \beta}[V] \\
V_{\alpha \beta i j} \equiv\left[V_{\alpha \beta i j}\right]-\left[A_{\alpha \beta i j}\right]+4 \partial_{\alpha \beta}\left[L_{i j}\right] \\
A_{\alpha \beta i j} \equiv\left[A_{\alpha \beta i j}\right]+4 \partial_{\alpha \beta}\left[L_{i j}\right],
\end{gathered}
$$

where we have adhered as closely as possible to the original notation. The fields within the square brackets are the original definitions that appear in reference 13. Strictly speaking, these same definitions ought to have been applied to the original $\mathrm{N}=2$, four dimensional expressions.

The flat limit "supersymmetry transformation laws" (analogous to (1) and (4)) can be found by combining the redefinitions above with results in [13]. For example, we find

$$
D_{\alpha i} \varphi=\frac{1}{2} \psi_{\alpha i}+\lambda_{\alpha i} \quad, \quad D_{\alpha i} \varphi_{j k}=C_{i(j \mid} \psi_{\alpha \mid k)}+2 \lambda_{\alpha i j k}
$$


It is simple exercise to write all such supersymmetry variations, so we forego repeating them all here. The flat limit component action when written in terms of the redefined fields above turns out to be,

$$
\begin{aligned}
\mathcal{S}_{\mathrm{HP}, \mathrm{N}=4}=\int d^{2} \sigma & \varphi \square \varphi+\varphi_{i j} \square \varphi^{i j}+i \psi^{\alpha i} \partial_{\alpha \beta} \bar{\psi}^{\beta}{ }_{i} \\
& +\left(\lambda^{\alpha i} \chi_{\alpha i}+\text { h.c. }\right)-2\left(\lambda^{\alpha i j k} \chi_{\alpha i j k}+\text { h.c. }\right) \\
& +\frac{1}{18} N \bar{N}+\frac{3}{8}\left(K^{i j} \bar{K}_{i j}-M^{i j} \bar{M}_{i j}\right)-\frac{5}{4} C^{i j k l} L_{i j k l} \\
& \left.-\frac{1}{36} G^{\alpha \beta} G_{\alpha \beta}-\frac{3}{8}\left(A^{\alpha \beta i j} A_{\alpha \beta i j}-V^{\alpha \beta i j} V_{\alpha \beta i j}\right)\right] .
\end{aligned}
$$

In this expression, we have written some of the auxiliary fields utilizing a matrix formulation. For example we note, (with $G_{\alpha \beta}=\left(G_{\beta \alpha}\right)^{*}$ as a consequence of the reduction from $4 \mathrm{D}$ )

$$
G_{\alpha \beta} \equiv C_{\alpha \beta} \mathcal{B}+i\left(\gamma^{3}\right)_{\alpha \beta} \mathcal{H}+\left(\gamma^{a}\right)_{\alpha \beta} \mathcal{V}_{a}
$$

for real fields $\mathcal{B}, \mathcal{H}$ and $\mathcal{V}_{a}$. Similar expressions follow for $V_{\alpha \beta i j}$ and $A_{\alpha \beta i j}$.

\section{$4 \quad$ An $\mathrm{N}=4$ Superstring Question?}

Some time ago, Pernici and van Nieuwenhuizen [5] (P-vN) gave what was purported to be an action for the $\mathrm{N}=4$ superstring. In fact it was stated [7] that our off-shell construction must describe this previous work. With the present investigation, however, it is seen that this cannot be the case! The models in reference [7] involving twisted theories and be cannot identified with the type of matter field representation used in reference [5]. Similarly, the HM-II N $=4$ superstring discussed in the last section also cannot be (at least not directly) identified with the $\mathrm{P}-\mathrm{vN}$ construction because the $\mathrm{SU}(2)$ under which the spin- $1 / 2$ fields form a doublet is here used to put the four spin- 0 fields into a $1 \mathrm{~s} 1 \mathrm{p}$ representation. The corresponding fields are all singlets in the P-vN work. Even more surprisingly, careful study of the P-vN theory shows that it is fundamentally different from any of the $\mathrm{N}=4$ models discussed in this paper!

The first sign of this difference can be noted by comparing the supergravity spectra

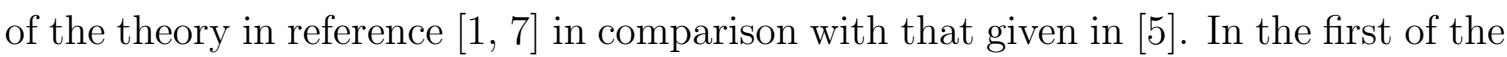
former, the $2 \mathrm{D}, \mathrm{N}=4$ supergravity pre-potential is identified as $V_{a i}{ }^{j}$ a Lorentz-vector $\mathrm{SU}(2)$-triplet superfield of engineering dimensions minus three. This determines the field content of conformal supergravity (as derived from superfield theory) to be $e_{a}{ }^{m}$, 
$\psi_{a}{ }^{\alpha i}$ and $B_{a}{ }_{i}{ }^{j}$. The multiplet contain a graviton, complex SU(2) doublet gravitini and the $\mathrm{SU}(2)$ gauge fields. By way of comparison, $\mathrm{P}-\mathrm{vN}$ have a supergravity spectrum that consist of $e_{a}{ }^{m}, \psi_{a}{ }^{\alpha \hat{A} i}$ and $B_{a}{ }_{i}{ }^{j}$ where their gravitini satisfy a Majorana condition. This may be regarded as simply a redefinition of the gravitini. However, this also means that the corresponding superspace description is based on a Majorana spinorial covariant derivative $\nabla_{\alpha \hat{A} i}$. Appropriate indices will thus appear in supertorsions.

In all of the $2 \mathrm{D}, \mathrm{N}=4$ superstrings described in superspace, the component level gravitini gauge covariant field strength must be locally $\mathrm{SU}(2)$ covariant. The corresponding quantities in the $\mathrm{P}-\mathrm{vN}$ construction violate this condition! Instead there one of the connections associated with the non-linear $\sigma$-model plays the role of $B_{a}{ }_{i}{ }^{j}$. Since this particular covariantization plays an absolutely critical role, it is not clear that the $\mathrm{P}-\mathrm{vN}$ theory can possess a limit where the $\sigma$-model manifold is flat and the supergravity fields are non-trivial! Closing this section, we note that we have not in this work addressed the more general question of the number of distinct local, $\mathrm{N}$ $=4 \sigma$-models. There are many such rigid models (see for example [12, 16]) that can act as the starting point of such investigations.

\section{Conclusions and Summary}

One of the amusing points now obvious is that we have, in our previous work [1, [], serendipitously discovered new $\mathrm{N}=4$ superstring theories! The original work on the $\mathrm{N}=4$ superstring [4] does not involve a twisted $\mathrm{N}=4$ supersymmetric matter multiplet. It is the on-shell truncation of a hypermultiplet $\mathrm{N}=4$ superstring that was considered in the first discussion of $\mathrm{N}=4$ superstrings. The most forceful way to see this is that the supersymmetry variation of the scalar fields in twisted multiplets always includes the appearance of the $\gamma^{3}$-matrix in at least one of the transformation laws. In the corresponding variations of the $2 \mathrm{D}, \mathrm{N}=4$ hypermultiplet, there is no appearance of the $\gamma^{3}$-matrix. Next we see that the twisted $\mathrm{N}=4$ superstrings are models that realize (off-shell) the $\mathrm{N}=4$ superconformal algebra in a minimal fashion on a total of 16-16 fields. Alternately, the HM-II, $\mathrm{N}=4$ superstring utilizes 40-40 component to realize (off-shell) the $\mathrm{N}=4$ superconformal algebra.

In a future work, we will discuss issue of the construction and characterization of superspace non-linear sigma models associated with these three different $\mathrm{N}=4$ superstring actions. There remains the study in detail of the local form of the component level actions and the explicit realization of superfield local conformal transformations. 
It also will be a topic of future study for us to apply a BV type of quantization technique to these models and study yet again the question of the critical dimension of $\mathrm{N}$ $=4$ superstring theory. As we now have complete off-shell structures for all of these theories, their quantization should follow as a straightforward exercise.

As we have seen, there is an unexpected embarass de richesse of off-shell $\mathrm{N}=4$ superstring theories. This is ironic since for over a decade there did not exist even one off-shell formulation of the $\mathrm{N}=4$ theory. In the following table, we summarize the features that distinguish each of the $\mathrm{N}=4$ superstrings.

\begin{tabular}{|c|c|c|c|}
\hline & TM - I & TM - II & HM - II \\
\hline \hline Spin - 0 SU(2) Rep. & $4 \mathrm{~s}$ & $1 \mathrm{~s} 1 \mathrm{p}$ & $1 \mathrm{~s} 1 \mathrm{p}$ \\
\hline Off - shell degrees & $16-16$ & $16-16$ & $40-40$ \\
\hline$\left(\gamma^{3}\right)$ in Q-trans. law & yes & yes & no \\
\hline
\end{tabular}

\section{Table I}

These features will be apparent when the currents for the $\mathrm{N}=4$ models are constructed using the techniques of superconformal field theory. The presence of $\left(\gamma^{3}\right)$ implies that the left and right currents do not appear in a totally isomorphic fashion.

Looking at the table above, also suggests that there exist one more $\mathrm{N}=4$ superstring that has four $\mathrm{SU}(2)$-singlet scalar fields and no $\left(\gamma^{3}\right)$ in its supersymmetry transformation laws among the physical scalars and spinors. The on-shell description of this multiplet is given by

$$
\begin{gathered}
D_{\alpha i} \mathcal{A}+C_{i j} \bar{D}_{\alpha}{ }^{j} \overline{\mathcal{B}}=\bar{D}_{\alpha}{ }^{i} \mathcal{A}=D_{\alpha i} \overline{\mathcal{B}}=0 \\
D_{\alpha i} \mathcal{A} \equiv \varphi_{\alpha i} \quad, \quad \bar{D}_{\alpha}{ }^{i} \overline{\mathcal{B}} \equiv C^{i j} \varphi_{\alpha j} \\
D_{\alpha i} \varphi_{\beta j}=i 2 C_{i j}\left(\gamma^{c}\right)_{\alpha \beta} \partial_{c} \overline{\mathcal{B}} \quad, \quad \bar{D}_{\alpha}{ }^{i} \varphi_{\beta j}=i 2 \delta_{i}{ }^{j}\left(\gamma^{c}\right)_{\alpha \beta} \partial_{c} \mathcal{A}
\end{gathered}
$$

The invariant action without auxiliary fields (on-shell action) is given by,

$$
\mathcal{S}_{\mathrm{HM}-\mathrm{I}, \mathrm{N}=4}=\int d^{2} \sigma\left[2 \overline{\mathcal{A}} \square \mathcal{A}+2 \overline{\mathcal{B}} \square \mathcal{B}+i \bar{\varphi}^{\alpha i}\left(\gamma^{c}\right)_{\alpha \beta} \partial_{c} \varphi^{\beta}{ }_{i}\right] .
$$

Attempting an off-shell formulation raises the whole issue of the "harmonic" hypermultiplet 15] which can be compactified to 2D. This model with its infinite number of auxiliary fields gives us the only known candidate for the off-shell description of the HM-I, $\mathrm{N}=4$ scalar multiplet. It is not known how to describe the off-shell representation with a finite number of auxiliary fields for this scalar multiplet. The 
real challenges are find out; (a.) if there is an alternate off-shell formulation of the HM-I scalar multiplet with a finite number of auxiliary fields and (b.) if the harmonic formulation of the HM-I scalar multiplet can be coupled to minimal 2D, $\mathrm{N}=$ 4 supergravity to provide an off-shell description to the HM-I, $\mathrm{N}=4$ superstring.

It is, perhaps, beneficial to use what we have learned and ask, "Are there any more $\mathrm{N}=4$ superstrings?" At this point, we do not know how to find the answer to this one way or the other. There are some possible variant representations of the hypermultiplet $\mathrm{N}=4$ superstring. In the original work [13], it was pointed out that the relaxed hypermultiplet could be formulated with slightly different sets of auxiliary fields. Compactification to $2 \mathrm{D}$ should preserve this possibility. So there is at least this ambiguity. We have no principle for ruling out further $\mathrm{N}=4$ superstrings.

The final interesting point raised by our title may have a resolution in terms of extending the notion of mirror symmetry to act between a hyper Kahler manifold and a quaternionic manifold. This is an exciting prospect. But for now we are simply left with the puzzle of our title.

\section{Acknowledgement}

S. J. G. wishes to acknowledge the hospitality of Drs. S. V. Ketov, O. Lechtenfeld and the Institut für Theoretische Physik of Universität Hannover extended during the period of this work. Useful comments from Martin Roček are also acknowledged. 


\section{References}

[1] S. James Gates, Jr., Liang Lu and R. Oerter, Phys. Lett. 218B (1989) 33.

[2] S. James Gates, Jr., C. M. Hull and M. Roček, Nucl. Phys. B248 (1984) 157.

[3] E. Kiritis, C. Kounnas and D. Lüst, Superstring Gravitational Wave Backgrounds with Spacetime Supersymmetry, preprint, CERN-TH.7218/94, HUB-IEP-94/5, LPTENS-94/10, hep-th/9404114.

[4] M. Admello, L. Brink, A. D'Adda, R. D'Auria, E. Napolitano, S. Sciuto, E. Del Giudice, P. Di Vecchia, S. Ferrara, F. Gliozzi, R. Musto, R. Pattorino, and J. Schwarz, Nucl. Phys. 114 (1976) 297.

[5] P. van Nieuwenhuizen, Int. J. Mod. Phys. A1, (1986), 155; M. Pernici and P. van Nieuwenhuizen, Phys. Lett. 169B (1986) 381.

[6] W. Siegel, Phys. Rev. Lett. 69 (1992) 1493; Phys. Rev. D46 (1992) 3235; ibid. D47 (1993) 2504, ibid. 2512.

[7] S. James Gates, Jr., Y. Hassoun and P. van Nieuwenhuizen, Nucl. Phys. B317 (1989) 302.

[8] R. Brooks and S. James Gates, Jr., Extended Supersymmetry and Super-BF Gauge Theory, M.I.T. preprint CTP-2339, UMD preprint UMDEPP 95-07.

[9] S. James Gates, Jr. and W. Siegel, Nucl. Phys. B187 (1981) 389.

[10] E.A. Ivanov and S.O. Krivonos, J. Phys. A17 (1984) L671; idem. Theor. Math. Phys. 63 (1985) 477.

[11] D.Z. Freedman and L. Alvarez-Gaumé, Commun. Math. Phys. 91 (1983) 87; S. James Gates, Jr., Nucl. Phys. B238 (1984) 349; A. Jourjine, Nucl. Phys. B236 (1984) 181.

[12] B.B. Deo and S. James Gates, Jr., Nucl. Phys. B254 (1985) 187.

[13] P.S. Howe, K.S.Stelle, and P.K. Townsend, Nucl. Phys. B214 (1983) 519; S.V.Ketov, J. Mod Phys. A3, (1988) 703; idem., Fort. der Physik, 36 (1988) 361. 
[14] P.S. Howe, K.S.Stelle, and P. West, Phys. Lett. 124B (1983) 55; P. West, in Proceedings of the Shelter Island II Conference on Quantum Field Theory and Fundamental Problems of Physics, eds. R.Jackiw, N.Khuri, S. Weinberg and E. Witten, M.I.T. Press (1993).

[15] A. Galperin, E. Ivanov, V. Ogievetsky and E. Sokatchev, JETP Lett. 40 (1984) 912; A. Galperin, E. Ivanov, S. Kalitzin, V. Ogievetsky and E. Sokatchev, Class. Quant. Grav. 1 (1984) 469.

[16] T. Buscher, U. Lindstrom and M. Roček, Phys. Lett. 202B (1988), U. Lindstrom and M. Roček, Commun. Math. Phys. 115 (1988) 21. 